\title{
COHOMOLOGICAL DIMENSION AND METRIZABLE SPACES
}

\author{
JERZY DYDAK
}

\begin{abstract}
The purpose of this paper is to address several problems posed by V. I. Kuzminov [Ku] regarding cohomological dimension of noncompact spaces. In particular, we prove the following results:
\end{abstract}

Theorem A. Suppose $X$ is metrizable and $G$ is the direct limit of the direct system $\left\{G_{s}, h_{s^{\prime}, s}, S\right\}$ of abelian groups. Then,

$$
\operatorname{dim}_{G} X \leq \max \left\{\operatorname{dim}_{G_{s}} X \mid s \in S\right\} .
$$

Theorem B. Let $X$ be a metrizable space and let $G$ be an abelian group. Let $l=\{p \mid p \cdot(G /$ Tor $G) \neq G /$ Tor $G\}$.
(a) If $G=\operatorname{Tor} G$, then $\operatorname{dim}_{G} X=\max \left\{\operatorname{dim}_{H} X \mid H \in \sigma(G)\right\}$,
(b) $\operatorname{dim}_{G} X=\max \left\{\operatorname{dim}_{\mathrm{Tor} G} X, \operatorname{dim}_{G / \operatorname{Tor} G} X\right\}$,
(c) $\operatorname{dim}_{G} X \geq \operatorname{dim}_{\mathbb{Q}} X$ if $G \neq$ Tor $G$,
(d) $\operatorname{dim}_{G} X \geq \operatorname{dim}_{\widehat{\mathbb{Z}}_{l}} X$, where $\widehat{\mathbb{Z}}_{l}$ is the group of l-adic integers,
(e) $\max \left(\operatorname{dim}_{G} X, \operatorname{dim}_{\mathbb{Q}} X+1\right) \geq \max \left\{\operatorname{dim}_{H} X \mid H \in \sigma(G)\right\}$,
(f) $\operatorname{dim}_{G} X \leq \operatorname{dim}_{\mathbf{Z}_{l}} X \leq \operatorname{dim}_{G} X+1$ if $G \neq 0$ is torsion-free.

Theorem B generalizes a well-known result of M. F. Bockstein [B].

\section{INTRODUCTION}

Of primary importance in cohomological dimension theory of compact spaces (see $[\mathrm{Ku}])$ are the following results of Bockstein $[\mathrm{B}]$ :

Bockstein Inequalities. For any compact Hausdorff space $X$ and any short exact sequence of abelian groups $0 \rightarrow G \rightarrow E \rightarrow \Pi \rightarrow 0$ the following inequalities hold:

(a) $\operatorname{dim}_{E} X \leq \max \left(\operatorname{dim}_{G} X, \operatorname{dim}_{\Pi} X\right)$,

(b) $\operatorname{dim}_{G} X \leq \max \left(\operatorname{dim}_{E} X, \operatorname{dim}_{\Pi} X+1\right)$,

(c) $\operatorname{dim}_{\Pi} X \leq \max \left(\operatorname{dim}_{E} X, \operatorname{dim}_{G} X-1\right)$.

In particular, for any prime $p$ :

(1) $\operatorname{dim}_{\mathbb{Z} / p^{\infty}} X \leq \operatorname{dim}_{\mathbb{Z} / p} X=\operatorname{dim}_{\mathbb{Z} / p^{k}} X \leq \operatorname{dim}_{\mathbb{Z} / p^{\infty}} X+1$,

(2) $\operatorname{dim}_{\mathbb{Q}} X \leq \operatorname{dim}_{\mathbb{Z}_{p}} X$,

(3) $\operatorname{dim}_{\mathbb{Z} / p} X \leq \operatorname{dim}_{\mathbb{Z}_{p}} X$,

Received by the editors February 1, 1991.

1980 Mathematics Subject Classification (1985 Revision). Primary 55M10; Secondary 54F45, $55 \mathrm{M} 15,55 \mathrm{U} 20$.

Key words and phrases. Cohomological dimension, absolute extensors, Eilenberg-Mac Lane complexes, metrizable spaces, $p$-adic integers.

Research supported in part by an NSF Grant DMS-9101283 and the Science Alliance of the University of Tennessee. 
(4) $\operatorname{dim}_{\mathbb{Z} / p^{\infty}} X \leq \max \left(\operatorname{dim}_{\mathbb{Q}} X, \operatorname{dim}_{\mathbb{Z}_{p}} X-1\right)$,

(5) $\operatorname{dim}_{\mathbb{Z}_{p}} X \leq \max \left(\operatorname{dim}_{\mathbb{Q}} X, \operatorname{dim}_{\mathbb{Z} / p^{\infty}} X+1\right)$.

Bockstein Theorem. For any abelian group $G$ and a compact Hausdorff space $X$,

$$
\operatorname{dim}_{G} X=\max \left\{\operatorname{dim}_{H} X \mid H \in \sigma(G)\right\} .
$$

Our notation is that of $\mathrm{D}$. Sullivan [Su]: Given a prime $p, \mathbb{Z} / p=\mathbb{Z} / p \mathbb{Z}$, $\mathbb{Z}_{p}=\{m / n \in \mathbb{Q} \mid n$ is not divisible by $p\} \quad(\mathbb{Q}$ being the rationals $)$ and $\mathbb{Z} / p^{\infty}=$ $\underset{\lim }{\longrightarrow}\left(\mathbb{Z} / p \rightarrow \mathbb{Z} / p^{2} \rightarrow \cdots \rightarrow \mathbb{Z} / p^{n} \rightarrow \cdots\right)$ is $\mathbb{Q} / \mathbb{Z}_{p}$. More generally, given any set $l$ of primes, $\mathbb{Z}_{l}=\{m / n \in \mathbb{Q} \mid n$ is not divisible by any $p \in l\}$ and $\mathbb{Z} / l^{\infty}:=\mathbb{Q} / \mathbb{Z}_{l}=\bigoplus_{p \in l} \mathbb{Z} / p^{\infty}$ (see [Su, p. 2.25]). In particular, $\mathbb{Z}_{\phi}=\mathbb{Q}$ and $\mathbb{Z}_{\{\text {all primes }}=\mathbb{Z}$.

The group $\widehat{\mathbb{Z}}_{p}$ of $p$-adic integers is the completion of $\mathbb{Z}$ in the topology generated by $p^{k} \cdot \mathbb{Z}, k=1, \ldots$, as the system of neighborhoods of 0 (see [Su, p. 1.18]). Similarly, there is a group of $l$-adic integers $\widehat{\mathbb{Z}}_{l}$ and $\widehat{\mathbb{Z}}_{l} \approx \prod_{p \in l} \widehat{\mathbb{Z}}_{p}$ (see [Ma, p. 475]).

Given an abelian group $G$ its Bockstein basis $\sigma(G)$ is a subset of $\{\mathbb{Q}\} \cup$ $\bigcup_{p}\left\{\mathbb{Z}_{p}, \mathbb{Z} / p, \mathbb{Z} / p^{\infty}\right\}$ ( $p$ is a prime) defined as follows:

(a) $\mathbb{Q} \in \sigma(G)$ iff $G / \operatorname{Tor} G \neq 0$ (Tor $G:=\{g \in G \mid m \cdot g=0$ for some $m \in \mathbb{Z}-\{0\}\})$,

(b) $\mathbb{Z}_{p} \in \sigma(G)$ iff $G /$ Tor $G$ is not divisible by $p$,

(c) $\mathbb{Z} / p \in \sigma(G)$ iff $p$-Tor $G$ is not divisible by $p \quad\left(p\right.$-Tor $G:=\left\{g \in G \mid p^{m}\right.$. $g=0$ for some $m \geq 1\}$ ),

(d) $\mathbb{Z} / p^{\infty} \in \sigma(G)$ iff $p$-Tor $G \neq 0$ is divisible by $p$.

Examples. (a) $\sigma(\mathbb{Z})=\{\mathbb{Q}\} \cup\left\{\mathbb{Z}_{p} \mid p\right.$ prime $\}$,

(b) $\sigma(\mathbb{Q})=\{\mathbb{Q}\}$,

(c) $\sigma\left(\mathbb{Z}_{p}\right)=\left\{\mathbb{Q}, \mathbb{Z}_{p}\right\}$,

(d) $\sigma(\mathbb{Z} / p)=\{\mathbb{Z} / p\}$

(e) $\sigma\left(\mathbb{Z} / p^{\infty}\right)=\left\{\mathbb{Z} / p^{\infty}\right\}$.

The proof of the Bockstein Theorem for compact spaces (see [Ku, pp. 1112]) relies heavily on the following two results (see [S, p. 246] and [W, p. 251] or [Ku, p. 31]):

\section{Universal Coefficient Formula.}

$$
0 \rightarrow \check{H}^{n}(X, A ; \mathbb{Z}) \otimes G \rightarrow \check{H}^{n}(X, A ; G) \rightarrow \check{H}^{n+1}(X, A ; \mathbb{Z}) * G \rightarrow 0
$$

is exact for any compact pair $(X, A)$ and any abelian group $G$.

Bockstein Exact Sequence. If $0 \rightarrow G \rightarrow E \rightarrow \Pi \rightarrow 0$ is a short exact sequence of abelian groups, then there is a natural exact sequence

$\cdots \rightarrow \check{H}^{n}(X, A ; G) \rightarrow \check{H}^{n}(X, A ; E) \rightarrow \check{H}^{n}(X, A ; \Pi) \rightarrow \check{H}^{n+1}(X, A ; G) \rightarrow \cdots$

for any paracompact space $X$ and its closed subspace $A$. Here $\check{H}^{n}(X, A ; G)$ stands for the nth Čech cohomology group of the pair $(X, A)$ with coefficients in $G$.

The reason Bockstein results are useful lies in the fact that $\mathbb{Q}, \mathbb{Z}_{p}$ and $\mathbb{Z} / p$ are principal ideal domains and there are certain geometrical constructions for 
$\mathbb{Q}, \mathbb{Z}_{p}$ and $\mathbb{Z} / p$ (see $\left[\mathrm{D}_{1}\right.$ and $\left.\mathrm{D}-\mathrm{W}_{3}\right]$ ) whose existence for arbitrary abelian groups is unknown.

While the Bockstein Inequalities are valid for paracompact spaces (they are a straightforward consequence of the Bockstein Exact Sequence-see [Ku]), the question of validity of Bockstein's Theorem in the realm of paracompact spaces is still open (compare [Ku, p. 32]).

Since the Universal Coefficient Formula is not known to be valid for noncompact spaces, one needs to develop entirely new techniques to be able to prove the Bockstein Theorem for noncompact spaces.

The purpose of this paper is to prove the following results (compare Theorem A with Theorem 19 in [Ku]):

Theorem A. Suppose $X$ is metrizable and $G$ is the direct limit of the direct system $\left\{G_{s}, h_{s^{\prime}, s}, S\right\}$ of abelian groups. Then

$$
\operatorname{dim}_{G} X \leq \max \left\{\operatorname{dim}_{G_{s}} X \mid s \in S\right\} .
$$

Theorem B. Let $X$ be a metrizable space and let $G$ be an abelian group. Let $l=\{p \mid p \cdot(G /$ Tor $G) \neq G /$ Tor $G\}$.

(a) If $G=\operatorname{Tor} G$, then $\operatorname{dim}_{G} X=\max \left\{\operatorname{dim}_{H} X \mid H \in \sigma(G)\right\}$,

(b) $\operatorname{dim}_{G} X=\max \left\{\operatorname{dim}_{\text {Tor } G} X, \operatorname{dim}_{G / \operatorname{Tor} G} X\right\}$,

(c) $\operatorname{dim}_{G} X \geq \operatorname{dim}_{\mathbb{Q}} X$ if $G \neq$ Tor $G$,

(d) $\operatorname{dim}_{G} X \geq \operatorname{dim}_{\widehat{\mathbb{Z}}_{l}} X$, where $\widehat{\mathbb{Z}}_{l}$ is the group of l-adic integers,

(e) $\max \left(\operatorname{dim}_{G} X, \operatorname{dim}_{\mathbb{Q}} X+1\right) \geq \max \left\{\operatorname{dim}_{H} X \mid H \in \sigma(G)\right\}$,

(f) $\operatorname{dim}_{G} X \leq \operatorname{dim}_{\mathbb{Z}_{1}} X \leq \operatorname{dim}_{G} X+1$, if $G \neq 0$ is torsion-free.

Remarks. The inequality $\operatorname{dim}_{G} X \geq \operatorname{dim}_{\text {Tor } G} X$ solves the second part of Problem 6 in [Ku] (asked for arbitrary paracompact spaces). Our proofs of both of the above theorems are valid for compactly generated paracompact spaces $X$ such that $\mathrm{CW}$ complexes are absolute neighborhood extensors of $X \times I$.

\section{ABSOLUTE EXTENSORS AND COHOMOLOGICAL DIMENSION}

2.1. Definition. A space $Y$ is an absolute (neighborhood) extensor of the space $X$ (notation: $Y \in A E(X)$ or $Y \in A N E(X)$ ) if every map $f: A \rightarrow Y, A$ closed in $X$, extends over $X$ (over a neighborhood $U$ of $A$ in $X$ ).

If $\mathscr{C}$ is a class of spaces, then $Y \in A E(\mathscr{C}) \quad(Y \in A N E(\mathscr{C}))$ means $Y \in$ $A E(X) \quad(Y \in A N E(\mathscr{C}))$ for all $X$ in $\mathscr{C}$.

2.2. Theorem (Y. Kodama [Ko]). $K \in A N E$ (metrizable spaces) for any $C W$ complex $K$.

2.3. Theorem. Suppose $p: E \rightarrow B$ is a map, $B$ is a regular cell complex and $X$ is a metrizable space such that $p^{-1}(\sigma) \in A E(X)$ for each cell $\sigma$ in $B$. If

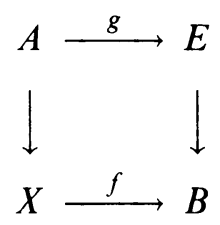

is a commutative diagram where $A$ is a closed subset of $X$ and $A \rightarrow X$ is the inclusion, then there is an extension $g: X \rightarrow E$ of $g$ and a homotopy $H: X \times I \rightarrow B$ joining $p \circ g^{\prime}$ and $f$ rel. $A$ so that $H\left(f^{-1}(\sigma) \times I\right) \subset \sigma$ for each 
cell $\sigma$ of $B$. Consequently, $E \in A E(X)$ if $B \in A E(X)$ and $B \in A E(X)$ if $E \in A E(X)$ and $B \in A N E(X \times I)$.

Proof. Recall that a regular cell complex (see [W, p. 81]) is a CW complex $B$ such that each closed $n$-cell $e$ of $B$ is homeomorphic to $\Delta^{n}$ and its boundary is the union of finitely many $(n-1)$-cells of $B$. Let $X_{n}=A \cup\{x \in X \mid f(x)$ lies in an $n$-cell of $B\}$ for each $n \geq-1$. We will construct by induction on $n \geq-1$ :

(1) a map $\alpha_{n} \in E^{X_{n}}$ which is an extension of $\alpha_{n-1}$ such that $\alpha_{n}\left(f^{-1}(\sigma)\right) \subset$ $p^{-1}(\sigma)$ and $\alpha_{n} \mid f^{-1}(\sigma)$ is continuous for each $k$-cell $\sigma$ of $B, k \leq n$,

(2) a homotopy $H_{n}: X_{n} \times I \rightarrow B$ (which is an extension of $H_{n-1}$ ) joining $p \circ \alpha_{n}$ and $f \mid X_{n}$ rel. $A$ so that $H_{n}\left(f^{-1}(\sigma) \times I\right) \subset \sigma$ and $H_{n}\left(f^{-1}(\sigma) \times I\right)$ is continuous for each $k$-cell $\sigma$ of $B, k \leq n$.

Notice that all the maps under construction are continuous. Indeed, if $H_{n}$ : $X_{n} \times I \rightarrow B$ is continuous and $H_{n+1}\left(f^{-1}(\sigma) \times I\right) \subset \sigma$ and $H_{n+1} \mid f^{-1}(\sigma) \times I$ is continuous for each $k$-cell $\sigma$ of $B, k \leq n+1$, then $H_{n+1} \mid f^{-1}(K) \times I$ is continuous for each finite subcomplex $K$ of $B$. Since $f(Z)$ is contained in a finite subcomplex of $B$ for any compact set $Z$ in $X, H_{n+1} \mid Z \times I$ is continuous. Thus, $H_{n+1}$ is continuous.

Put $\alpha_{-1}=g$ and $H_{-1}(x, t)=f(x)$ for $(x, t) \in A \times I$. Given $\alpha_{n}, H_{n}$ and an $(n+1)$-cell $\sigma$ of $B$ we define $\alpha_{n+1} \mid f^{-1}(\sigma)$ as an extension of

$$
\alpha_{n} \mid f^{-1}(\partial \sigma): f^{-1}(\partial \sigma) \rightarrow p^{-1}(\sigma) \in A E(X) .
$$

$H_{n+1} \mid f^{-1}(\sigma) \times I$ is an extension of $F: f^{-1}(\sigma) \times\{0,1\} \cup f^{-1}(\partial \sigma) \times I \rightarrow \sigma$ defined by $F\left|f^{-1}(\sigma) \times\{0\}=p \circ \alpha_{n+1}, \quad F\right| f^{-1}(\sigma) \times\{1\}=f \mid f^{-1}(\sigma)$, and $F\left|f^{-1}(\partial \sigma) \times I=H_{n}\right| f^{-1}(\partial \sigma) \times I$. This induces $\alpha_{n} \in E^{X_{n+1}}$ and $H_{n+1}$. The family $\left\{\alpha_{n}\right\}_{n \geq-1}$ induces $g^{\prime} \in E^{X}$ and $\left\{H_{n}\right\}_{n \geq-1}$ induces $H$.

Assume $B \in A E(X)$. Suppose $g: A \rightarrow E$ is a map. Choose an extension $f: X \rightarrow B$ of $p \circ g: A \rightarrow B$ and use the first part of Theorem 2.3 to produce an extension of $g$. Thus, $E \in A E(X)$.

Assume $E \in A E(X)$. Suppose $g: C \rightarrow B$ is a map, $C$ a closed subset of $X$. Thus, using the first part of Theorem 2.3, we have a map $\alpha: C \rightarrow E$ such that $p \circ \alpha$ and $g$ are homotopic. Choose an extension $\alpha^{\prime}: X \rightarrow E$. Then $p \circ \alpha^{\prime}$ is a homotopy extension of $g$, so the Homotopy Extension Theorem (in view of $B \in A N E(X \times I))$ implies that $g$ extends over $X$. Thus, $B \in A E(X)$.

Remark. Theorem 2.3 is based on an idea due to S. Ferry (see [Wa, Appendix A]).

2.4. Definition. For a paracompact space $X$ and an abelian group $G$ we define the cohomological dimension $\operatorname{dim}_{G} X$ of $X$ with coefficient group $G$ as

$\min \{n:$ there is a space $Y \in A E(X) \cap A N E$ (paracompact) $\cap K(G, n)\}$.

Here, $K(G, n)$ stands for the class of spaces homotopy equivalent to a $\mathrm{CW}$ complex $K$ such that $\pi_{n} K \approx G$ and $\pi_{i} K=0$ for $i \neq n$ (see [W and $\mathrm{Ku}$ ]).

2.5. Theorem. For a metrizable space $X$ and an abelian group $G$ the following conditions are equivalent:

(1) $\operatorname{dim}_{G} X \leq n$,

(2) $\check{H}^{n+1}(X, A ; G)=0$ for all closed subsets $A$ of $X$, 

$X$.

(3) $\check{H}^{n}(X ; G) \rightarrow \check{H}^{n}(A ; G)$ is an epimorphism for all closed subsets $A$ of

Proof. (1) $\rightarrow$ (2). Choose a simplicial complex $K \in K(G, n+1)$ (equipped with the Whitehead topology) with basepoint $*$. Let $P$ be the space of paths in $K$ starting at $* . \pi: P \rightarrow K$ is defined by $\pi(\omega)=\omega(1)$. Notice that $\pi$ is a Hurewicz fibration (see [W, p. 42]) and $\pi^{-1}(*)=\Omega K \in K(G, n)$ (see [M]). Therefore $\pi^{-1}(\Delta) \in K(G, n)$ for each simplex $\Delta$ of $K$. Also, one can check directly that $\pi^{-1}(\Delta) \in A N E$ (metrizable spaces): map $g: B \rightarrow \pi^{-1}(\Delta)$, $B$ being a closed subset of a metrizable space $Y$, corresponds to a homotopy $H: B \times I \rightarrow K$ such that $H(B \times\{0\})=\{*\}$ and $H(B \times\{1\}) \subset \Delta$. Therefore, it extends to $G: Y \times\{0,1\} \cup B \times I \rightarrow K$, so that $G(Y \times\{0\})=\{*\}$ and $G(Y \times\{1\}) \subset \Delta$. Finally, $G$ extends to a neighborhood of $Y \times\{0,1\} \cup B \times I$ in $Y \times I$ which contains $U \times I$ for some neighborhood $U$ of $B$ in $Y$. Thus, $\pi^{-1}(\Delta) \in A E(X)$ for each simplex $\Delta$ of $K$.

Suppose $f:(X, A) \rightarrow(K, *)$. By Theorem 2.3 there is $g:(X, A) \rightarrow(P$, constant path) such that $\pi \circ g \approx f$ rel. $A$. Thus $f \approx 0 \quad(P$ is contractible).

$(2) \rightarrow(3)$. Follows from the Long Exact Sequence

$$
\cdots \check{H}^{n-1}(A) \rightarrow \check{H}^{n}(X, A) \rightarrow \check{H}^{n}(X) \rightarrow \check{H}^{n}(A) \rightarrow \check{H}^{n+1}(X, A) \cdots .
$$

$(3) \rightarrow(1)$. Follows from the Homotopy Extension Theorem.

Remark. Theorem 2.5 was proved by $\mathrm{H}$. Cohen [C] for locally compact spaces and by Skljarenko (see [Ku]) for paracompact spaces.

Theorem A is an easy corollary to Theorem 2.3 and the following:

2.6. Lemma. Suppose $\left\{G_{s}, h_{s^{\prime}, s}, S\right\}$ is a direct system of abelian groups. Let $N$ be the simplicial complex whose set of vertices is $S$ and $\left\langle s_{0} \cdots s_{m}\right\rangle$ is an $m$-simplex in $N$ iff $s_{0}>\cdots>s_{m}$. Then

(a) $N$ is contractible,

(b) there is a $C W$ complex $L$ of the type $K\left(\lim _{\longrightarrow}\left\{G_{s}, h_{s^{\prime}, s}, S\right\}, n\right)$ and a map $\pi: L \rightarrow N$ such that $\pi^{-1}(\Delta)$ is a subcomplex of $L$ of the type $K\left(G_{s}, n\right)$ for each simplex $\Delta$ of $N$ (here $s$ is the maximal vertex of $\Delta$ ). Moreover, the inclusion $\pi^{-1}\left(\Delta^{\prime}\right) \rightarrow \pi^{-1}(\Delta)$ induces $h_{s^{\prime}, s}$ on the nth homotopy groups if $\Delta^{\prime} \subset \Delta$.

Proof. (a) Given any finite subcomplex $N^{\prime}$ of $N$ choose $s \in S$ such that $s>v$ for each vertex $v$ of $N^{\prime}$. Now, it is clear that $s$ serves as the vertex of a cone over $N^{\prime}$ in $N$. Thus, the inclusion $N^{\prime} \rightarrow N$ is homotopically trivial and $N$ is contractible.

(b) Given $s \in S$, let $X(s)=G_{s} \times S^{n} / G_{s} \times\{*\}$ be the wedge of $n$-spheres. Here * is the basepoint of $S^{n}$ and there are $\left|G_{s}\right|$ (cardinality of $G_{s}$ ) spheres in the wedge. Points of $X(s)$ will be denoted by $(g, x)$ with the convention $(g, *)=(h, *)$ for all $g, h \in G_{s}$. Notice that $\pi_{n} X(s)$ is the free (abelian if $n \geq 2)$ group $F\left(G_{s}\right)$ generated by $G_{s}$ (as a set). If $s^{\prime}<s$ let $f_{s^{\prime}, s}: X\left(s^{\prime}\right) \rightarrow$ $X(s)$ be the map defined by $f_{s^{\prime}}, s(g, x)=\left(h_{s^{\prime}, s}(g), x\right)$ for $(g, x) \in X\left(s^{\prime}\right)$. Given a simplex $\Delta=\left\langle s_{0} \cdots s_{m}\right\rangle$ in $N$, where $\left.s_{0}>\cdots\right\rangle s_{m}$, let $s_{0}$ be denoted by $\max (\Delta)$ and let $s_{m}$ be denoted by $\min (\Delta)$. We are going to construct, by induction on $\operatorname{dim} \Delta, \mathrm{CW}$ complexes $X(\Delta)$ and maps $\pi(\Delta): X(\Delta) \rightarrow \Delta$, $p(\Delta): X(\max (\Delta)) \times \Delta \rightarrow X(\Delta)$ such that the following conditions are satisfied:

(1) $X(\Delta)=X(s)$ if $\Delta=\langle s\rangle$ 
(2) $X\left(\Delta^{\prime}\right) \subset X(\Delta)$ if $\Delta^{\prime}$ is a face of $\Delta$,

(3) the inclusion $X(\max (\Delta)) \rightarrow X(\Delta)$ is a homotopy equivalence,

(4) $\pi(\Delta) \mid X\left(\Delta^{\prime}\right)=\pi\left(\Delta^{\prime}\right)$ if $\Delta^{\prime} \subset \Delta$.

Suppose $\pi(\Delta): X(\Delta) \rightarrow \Delta$ and $p(\Delta): X(\max (\Delta)) \times \Delta \rightarrow X(\Delta)$ are defined for all $\Delta$ with $\operatorname{dim} \Delta<k, k \geq 1$. Given $\Delta$ with $\operatorname{dim} \Delta=k, X(\Delta)$ is defined as $(X(\max (\Delta)) \times \Delta) \cup_{\alpha}\left(\bigcup\left\{X\left(\Delta^{\prime}\right) \mid \Delta^{\prime} \subset \Delta\right\}\right)$, where $\alpha: X(\max (\Delta)) \times$ $(\partial \Delta) \rightarrow\left(\bigcup\left\{X\left(\Delta^{\prime}\right) \mid \Delta^{\prime} \subset \Delta\right\}\right)$ is defined as follows: on $X(\max (\Delta)) \times \Delta^{\prime}, \alpha$ is the composition of $f_{\max (\Delta), \max \left(\Delta^{\prime}\right)} \times$ id: $X(\max (\Delta)) \times \Delta^{\prime} \rightarrow X\left(\max \left(\Delta^{\prime}\right)\right) \times \Delta^{\prime}$ and $p\left(\Delta^{\prime}\right): X\left(\max \left(\Delta^{\prime}\right)\right) \times \Delta^{\prime} \rightarrow X\left(\Delta^{\prime}\right)$. Define $p(\Delta): X(\max (\Delta)) \times \Delta \rightarrow X(\Delta)$ as the projection. In view of condition (4) there is $\pi(\Delta): X(\Delta) \rightarrow \Delta$.

Notice that there is $\pi: X=\bigcup X(\Delta) \rightarrow N$ and $X$ contains

$$
N=\bigcup p(\Delta)(\{*\} \times \Delta)
$$

so that $\pi \mid N: N \rightarrow N$ is the identity. Therefore, when computing the homotopy groups of $X$, we may replace $X$ by $X / N$ (in view of contractibility of $N$ ). The CW complex $L$ is constructed as follows: $L=\bigcup L(\Delta)$, where $L(\Delta)$ contains $X(\Delta)$ as a subcomplex and $\pi: L \rightarrow N$ is an extension of $\pi: X \rightarrow N$. First of all, $L(s)$ is obtained from $X(s)$ by attaching $(n+1)$-cells to kill the kernel of $F\left(G_{s}\right) \rightarrow G_{s}$ and by attaching $(n+i)$-cells, $i \geq 2$, to kill higher homotopy groups of $X(s)$. We send $L(s)$ to $s$ via $\pi$. Assume $L(\Delta)$ is defined for all $\Delta$ with $\operatorname{dim} \Delta<k, k \geq 1$. If $\operatorname{dim} \Delta=k$, consider $X(\Delta) \cup \bigcup\left\{L\left(\Delta^{\prime}\right) \mid \Delta^{\prime} \subset \Delta\right\}$. It contains $X(\Delta) \cup L(\max (\Delta))$ which contains $L(\max (\Delta))$ as a deformation retract. The $(n+1)$-cells contained in

$$
X(\Delta) \cup \bigcup\left\{L\left(\Delta^{\prime}\right) \mid \Delta^{\prime} \subset \Delta\right\}-(X(\Delta) \cup L(\max (\Delta)))
$$

are attached along maps which are null-homotopic in $X(\Delta) \cup L(\max (\Delta))$. Therefore, the $n$th homotopy group of $X(\Delta) \cup \bigcup\left\{L\left(\Delta^{\prime}\right) \mid \Delta^{\prime} \subset \Delta\right\}$ is $G_{\max (\Delta)} \cdot L(\Delta)$ is formed from $X(\Delta) \cup \bigcup\left\{L\left(\Delta^{\prime}\right) \mid \Delta^{\prime} \subset \Delta\right\}$ by attaching $(n+i)$-cells, $i \geq 2$, to kill higher homotopy groups. Once an open cell $e$ is attached to $X(\Delta) \cup$ $\bigcup\left\{L\left(\Delta^{\prime}\right) \mid \Delta^{\prime} \subset \Delta\right\}$, the map $\pi$ is extended over $e$ so that $\pi(e) \subset$ int $\Delta$.

Proof of Theorem A. Suppose $n=\max \left\{\operatorname{dim}_{G_{s}} X \mid s \in S\right\}$. Choose a CW complex $L$ of the type $K\left(\lim \left\{G_{s}, h_{s^{\prime}, s}, S\right\}, n\right)$ and a map $\pi: L \rightarrow N$ such that $\pi^{-1}(\Delta)$ is a subcomplex of $L$ of the type $K\left(G_{s}, n\right)$ for each simplex $\Delta$ of $N$ (here $s$ is the maximal vertex of $\Delta)$. Thus, $\pi^{-1}(\Delta) \in A E(X)$ for each simplex $\Delta$ of $N$ and, since $N \in A E(X)$ ( $N$ is contractible), $L \in A E(X)$ by 2.3.

2.7. Corollary. Suppose $X$ is metrizable. If $G=\bigoplus_{s \in S} G_{s}$, then

$$
\operatorname{dim}_{G} X=\max \left\{\operatorname{dim}_{G_{s}} X \mid s \in S\right\} .
$$

Proof. Corollary 2.7 holds if $S$ is finite as $\prod_{s \in S} K_{s}$ is an

$$
A E(X) \cap K\left(\bigoplus_{s \in S} G_{s}, n\right)
$$

if each $K_{s} \in A E(X) \cap K\left(G_{s}, n\right)$. If $S$ is arbitrary, then $\bigoplus_{s \in S} G_{s}$ is a direct limit of $\bigoplus_{s \in T} G_{s}$, where $T$ varies through all finite subsets of $S$.

2.8. Corollary. Suppose $X$ is metrizable and $M$ is a torsion-free $R$-module, $R$ being both a principal ideal domain and an integral domain. Then

$$
\operatorname{dim}_{M \otimes_{R} N} X \leq \operatorname{dim}_{M} X
$$


for any $R$-module $N$. In particular,

$$
\operatorname{dim}_{N} X \leq \operatorname{dim}_{R} X
$$

for any $R$-module $N$.

Proof. By Corollary 2.7, Corollary 2.8 holds if $N$ is a free $R$-module. From the exactness of $0 \rightarrow R \rightarrow R \rightarrow R / m \cdot R \rightarrow 0$ one gets exactness of $0 \rightarrow M=$ $M \otimes_{R} R \rightarrow M \otimes_{R} R \rightarrow M \otimes_{R}(R / m \cdot R) \rightarrow 0$ and Bockstein Inequality (c) implies $\operatorname{dim}_{M \otimes_{R}(R / m \cdot R)} X \leq \operatorname{dim}_{M} X$. By Corollary 2.7, $\operatorname{dim}_{M \otimes_{R} N} X \leq \operatorname{dim}_{M} X$ for each finitely generated $R$-module $N$. Since every $R$-module is a direct limit of its finitely generated submodules and the torsion product commutes with taking the direct limit, Corollary 2.8 follows.

2.9. Corollary. Suppose $X$ is metrizable and $G=\prod_{s \in S} G_{s}$ is the direct product of torsion-free abelian groups $\left\{G_{s}\right\}_{s \in S}$ such that for each prime number $p$ the set $\left\{s \in S \mid p \cdot G_{s} \neq G_{s}\right\}$ is finite. Then

$$
\operatorname{dim}_{G} X=\max \left\{\operatorname{dim}_{G_{s}} X \mid s \in S\right\} .
$$

Proof. Clearly, $\operatorname{dim}_{G} X \geq \max \left\{\operatorname{dim}_{G_{s}} X \mid s \in S\right\}$ as each $G_{s}$ is a direct summand of $G$. Suppose $\max \left\{\operatorname{dim}_{G_{s}} X \mid s \in S\right\}=n$. Notice that $H=G / G^{\prime}$, $\left(G^{\prime}=\bigoplus_{s \in S} G_{s}\right)$ is torsion-free and divisible. Hence, $H$ is a direct sum of copies of $\mathbb{Q}$. Since $\operatorname{dim}_{\mathbb{Q}} X=\operatorname{dim}_{H} X=\operatorname{dim}_{G^{\prime} \otimes \mathbb{Q}} X \leq n$, the exact sequence $0 \rightarrow G^{\prime} \rightarrow G \rightarrow H \rightarrow 0$ implies $\operatorname{dim}_{G} X \leq n$.

2.10. Corollary. If $X$ is compact Hausdorff and $G$ is a torsion-free abelian group, then for any map $f: X \rightarrow K(G, n)$ there is a finitely generated free subgroup $F$ of $G$ such that $f$ factors up to homotopy as $X \rightarrow K(F, n) \rightarrow K(G, n)$. Consequently, if $0 \rightarrow G^{\prime \prime} \rightarrow G^{\prime} \rightarrow G \rightarrow 0$ is exact, then $\check{H}^{n}\left(X, A ; G^{\prime}\right) \rightarrow$ $\check{H}^{n}(X, A ; G)$ is an epimorphism and

$$
\operatorname{dim}_{G^{\prime}} X=\max \left(\operatorname{dim}_{G} X, \operatorname{dim}_{G^{\prime \prime}} X\right) .
$$

Proof. $G$ is the direct limit of $\left\{G_{s}, h_{s^{\prime}, s}, S\right\}$, where each $G_{s}$ is a finitely generated free subgroup of $G$ and each $h_{s^{\prime}, s}$ is an inclusion. Choose $\pi: L \rightarrow N$ as in Lemma 2.6. If $f:(X, A) \rightarrow L$ is a map, $\pi \circ f(X)$ is contained in a finite subcomplex $N^{\prime}$ of $N$. Choose $s \in S$ such that $s>v$ for each vertex $v$ of $N^{\prime}$. Now, it is clear that $s$ serves as the vertex of a cone over $N^{\prime}$ in $N$. Also, notice that $\pi^{-1}(s) \rightarrow \pi^{-1}\left(N^{\prime}\right)$ is a homotopy equivalence. Thus, $f$ factors through $\pi^{-1}\left(N^{\prime}\right) \in K\left(G_{s}, n\right) . \check{H}^{n}\left(X, A ; G^{\prime}\right) \rightarrow \check{H}^{n}(X, A ; G)$ being an epimorphism follows from the fact that any homomorphism $F \rightarrow G, F$ being free, can be factored as $F \rightarrow G^{\prime} \rightarrow G$. Finally, $\operatorname{dim}_{G^{\prime}} X=\max \left(\operatorname{dim}_{G} X, \operatorname{dim}_{G^{\prime \prime}} X\right)$ follows from the Bockstein Exact Sequence

$$
\begin{aligned}
\cdots & \rightarrow \check{H}^{n+1}\left(X, A ; G^{\prime \prime}\right) \rightarrow \check{H}^{n+1}\left(X, A ; G^{\prime}\right) \\
& \rightarrow \check{H}^{n+1}(X, A ; G) \rightarrow \check{H}^{n+2}\left(X, A ; G^{\prime \prime}\right) \rightarrow \cdots
\end{aligned}
$$

and Theorem 2.5.

Remark. The second part of Corollary 2.10 is typically proved using the Universal Coefficient Formula (see [Ku]).

\section{Proof of Theorem B}

(a) Suppose $G=\operatorname{Tor} G=\bigoplus_{p} p$-Tor $G$. To show $\operatorname{dim}_{G} X=\max \left\{\operatorname{dim}_{H} X \mid\right.$ $H \in \sigma(G)\}$ it suffices to prove $\operatorname{dim}_{G} X=\max \left\{\operatorname{dim}_{H} X \mid H \in \sigma(G)\right\}$ for any group $G=p$-Tor $G$. 
If $\mathbb{Z} / p \in \sigma(G)$, then $G$ is not equal to $p \cdot G$, so (see [Sz]) $\mathbb{Z} / p^{k}$ is a direct summand of $G$ for some $k \geq 1$. Thus $\operatorname{dim}_{G} X \geq \operatorname{dim}_{\mathbb{Z} / p^{k}} X=\operatorname{dim}_{\mathbb{Z} / p} X=$ $\max \left\{\operatorname{dim}_{H} X \mid H \in \sigma(G)\right\}$. On the other hand, $G=p$-Tor $G$ is a direct limit of its finite subgroups which are direct sums of groups isomorphic to $\mathbb{Z} / p^{m}$ for some $m$. Thus, $\operatorname{dim}_{G} X \leq \operatorname{dim}_{\mathbb{Z} / p} X=\max \left\{\operatorname{dim}_{H} X \mid H \in \sigma(G)\right\}$ by Theorem A.

If $\mathbb{Z} / p^{\infty} \in \sigma(G)$, then $G$ is equal to $p \cdot G$, so $G$ is isomorphic to a direct sum of copies of $\mathbb{Z} / p^{\infty}$ (see [F, Chapter 4]). Thus, $\operatorname{dim}_{G} X=\operatorname{dim}_{\mathbb{Z} / p^{\infty}} X=$ $\max \left\{\operatorname{dim}_{H} X \mid H \in \sigma(G)\right\}$ by Corollary 2.7 .

(b) In view of exactness of $0 \rightarrow \operatorname{Tor} G \rightarrow G \rightarrow G /$ Tor $G \rightarrow 0$ we have

$$
\operatorname{dim}_{G / \operatorname{Tor} G} X \leq \max \left(\operatorname{dim}_{G} X, \operatorname{dim}_{\text {Tor } G} X-1\right)
$$

and

$$
\operatorname{dim}_{G} X \leq \max \left(\operatorname{dim}_{G / \text { Tor } G} X, \operatorname{dim}_{\text {Tor } G} X\right)
$$

(see Bockstein Inequalities (a), (c)). So, it suffices to prove $\operatorname{dim}_{G} X \geq \operatorname{dim}_{\text {Tor } G} X$. If $\mathbb{Z} / p \in \sigma(G)$, then $\mathbb{Z} / p^{k}$ is a direct summand of $G$ (see [Sz]) for some $k \geq 1$. Thus, $\operatorname{dim}_{G} X \geq \operatorname{dim}_{\mathbb{Z} / p} X$. If $\mathbb{Z} / p^{\infty} \in \sigma(G)$, then $\mathbb{Z} / p^{\infty}$ is a direct summand of $G$ by [F, Chapter 4]. Thus, $\operatorname{dim}_{G} X \geq \operatorname{dim}_{\mathbb{Z} / p^{\infty}} X$. In view of (a), $\operatorname{dim}_{G} X \geq \operatorname{dim}_{\text {Tor } G} X$.

(c) Let $\operatorname{dim}_{G} X=n$. By Corollary $2.8, \operatorname{dim}_{G \otimes \mathbb{Q}} X \leq n$. Since $G \otimes \mathbb{Q}$ contains $\mathbb{Q}$ as a direct summand (it is a vector space over $\mathbb{Q}$ ), $\operatorname{dim}_{\mathbb{Q}} X \leq n$.

(d) Let $\operatorname{dim}_{G} X=n$. We may assume Tor $G=0$ (replace $G$ by $G /$ Tor $G$ if necessary). Suppose $p$ is a prime and $p \cdot G \neq G$. Put $G^{\prime}:=G \otimes \mathbb{Z}_{p}$. By Corollary 2.8, $\operatorname{dim}_{G^{\prime}} X \leq n$ and $\operatorname{dim}_{G^{\prime} \otimes \widehat{\mathbb{Z}}_{p}} X \leq n$. Also, $G^{\prime}$ is not divisible by $p$, so choose $g \in G^{\prime}$ which is not divisible by $p$. Notice that $G^{\prime} / \mathbb{Z}_{p} \cdot g$ is torsion-free. Indeed, if $k \cdot x=\frac{m}{n} \cdot g, k, m, n$ being integers and $\frac{m}{n} \in \mathbb{Z}_{p}$, then we may assume that one of $k, m$ is not divisible by $p$. If $k$ is not divisible by $p$, then $x=\frac{m}{n k} \cdot g \in \mathbb{Z}_{p} \cdot g$. If $k$ is divisible by $p$, then $g=\frac{n k}{m} \cdot x$ is divisible by $p$, a contradiction. By tensoring $0 \rightarrow \mathbb{Z}_{p} \cdot g \rightarrow G^{\prime} \rightarrow G^{\prime} / \mathbb{Z}_{p} \cdot g \rightarrow 0$ with $\widehat{\mathbb{Z}}_{p}$ we get $0 \rightarrow\left(\mathbb{Z}_{p} \cdot g\right) \otimes \widehat{\mathbb{Z}}_{p} \rightarrow G^{\prime} \otimes \widehat{\mathbb{Z}}_{p} \rightarrow\left(G^{\prime} / \mathbb{Z}_{p} \cdot g\right) \otimes \widehat{\mathbb{Z}}_{p} \rightarrow 0$. Since $\left(\mathbb{Z}_{p} \cdot g\right) \otimes \widehat{\mathbb{Z}}_{p} \approx \widehat{\mathbb{Z}}_{p}$ is algebraically compact and $\left(G^{\prime} / \mathbb{Z}_{p} \cdot g\right) \otimes \widehat{\mathbb{Z}}_{p}$ is torsion-free, $\left(\mathbb{Z}_{p} \cdot g\right) \otimes \widehat{\mathbb{Z}}_{p}$ is a direct summand of $G^{\prime} \otimes \widehat{\mathbb{Z}}_{p}$ (see [F, Proposition 39.4]). Thus, $\operatorname{dim}_{\widehat{\mathbb{Z}}_{p}} X \leq n$ and $\operatorname{dim}_{\widehat{\mathbb{Z}}_{l}} X \leq n$ by Corollary 2.9.

(e) In view of (a) and (b) it suffices to consider the case $H=\mathbb{Z}_{p}$. From the exactness of $0 \rightarrow \mathbb{Z}_{p} \rightarrow \widehat{\mathbb{Z}}_{p} \rightarrow \widehat{\mathbb{Z}}_{p} / \mathbb{Z}_{p} \rightarrow 0$, we get (using Bockstein Inequality (b)),

$$
\operatorname{dim}_{\mathbb{Z}_{p}} X \leq \max \left(\operatorname{dim}_{\widehat{\mathbb{Z}}_{p}} X, \operatorname{dim}_{\widehat{\mathbb{Z}}_{p} / \mathbb{Z}_{p}} X+1\right) .
$$

Since $\widehat{\mathbb{Z}}_{p} / \mathbb{Z}_{p}$ is a vector space over $\mathbb{Q}, \operatorname{dim}_{\widehat{\mathbb{Z}}_{p} / \mathbb{Z}_{p}} X=\operatorname{dim}_{\mathbb{Q}} X$ by Corollary 2.7 . Thus,

$$
\max \left(\operatorname{dim}_{G} X, \operatorname{dim}_{\mathbb{Q}} X+1\right) \geq \max \left\{\operatorname{dim}_{H} X \mid H \in \sigma(G)\right\} .
$$

(f) Let $\operatorname{dim}_{\mathbb{Z}_{l}} X=n$. Notice that $G$ is a $\mathbb{Z}_{l}$-module. Thus, $\operatorname{dim}_{G} X \leq n$ by Corollary 2.8. If $p \in l$, then $\operatorname{dim}_{G \otimes(\mathbb{Z} / p)} X \leq \operatorname{dim}_{G} X$ by Corollary 2.8. Since $G \otimes(\mathbb{Z} / p) \neq 0$ and $\sigma(G \otimes(\mathbb{Z} / p))=\{\mathbb{Z} / p\}$ (all the elements in $G \otimes(\mathbb{Z} / p)$ are of order $p$, so the group cannot be divisible by $p), \operatorname{dim}_{\mathbb{Z} / p^{\infty}} X \leq \operatorname{dim}_{\mathbb{Z} / p} X=$ $\operatorname{dim}_{G \otimes(\mathbb{Z} / p)} X \leq \operatorname{dim}_{G} X$. From the exactness of $0 \rightarrow \mathbb{Z}_{l} \rightarrow \mathbb{Q} \rightarrow \bigoplus_{p \in l} \mathbb{Z} / p^{\infty} \rightarrow$ 
0 , we conclude (see Bockstein Inequality (b)),

$$
\operatorname{dim}_{\mathbb{Z}_{l}} X \leq \max \left(\operatorname{dim}_{\mathbb{Q}} X, \operatorname{dim}_{\oplus_{p \in l} \mathbb{Z} / p^{\infty}} X+1\right) \leq \operatorname{dim}_{G} X+1 .
$$

3.1. Corollary. Suppose $X$ is metrizable. Then

$$
\operatorname{dim}_{G \otimes H} X \leq \operatorname{dim}_{G} X
$$

for any abelian groups $G$ and $H$.

Proof. If $G$ is torsion-free it follows from Corollary 2.8. If $G=p$-Tor $G$, then $\sigma$ (image of $G \otimes H) \subset \sigma(G)$. Indeed, $\mathbb{Z} / p^{\infty} \in \sigma(G)$ iff $G$ is a divisible $p$-group and both operations of taking torsion product and taking the quotient group preserve divisibility. Thus, $\sigma((G \otimes H) / A) \subset \sigma(G)$ for any torsion group $G$ and any subgroup $A$ of $G \otimes H$. From the exactness of (Tor $G) \otimes H \rightarrow G \otimes H \rightarrow$ $(G /$ Tor $G) \otimes H \rightarrow 0$ we get $0 \rightarrow(($ Tor $G) \otimes H) / A \rightarrow G \otimes H \rightarrow(G /$ Tor $G) \otimes H \rightarrow$ 0 , where $A=\operatorname{ker}((\operatorname{Tor} G) \otimes H \rightarrow G \otimes H)$, and by Theorem B(ab),

$$
\operatorname{dim}_{G \otimes H} X \leq \max \left(\operatorname{dim}_{(\operatorname{Tor} G) \otimes H} X, \operatorname{dim}_{(G / \text { Tor } G) \otimes H} X\right) \leq \operatorname{dim}_{G} X .
$$

\section{Applications}

Theorem C. Suppose $X=A \cup B$ is metrizable and $G$ is an abelian group. Then,

$$
\operatorname{dim}_{G} X \leq \max \left(\operatorname{dim}_{G} A, 2\right)+\max \left(\operatorname{dim}_{G} B, 2\right)+3 .
$$

Proof. Our goal is to show $\operatorname{dim}_{H} X \leq \max \left(\operatorname{dim}_{G} A, 2\right)+\max \left(\operatorname{dim}_{G} B, 2\right)+3$ for all $H \in \sigma(G) \cup\left\{\mathbb{Z}_{l}\right\}$ and then apply Theorem $\mathrm{B}(\mathrm{b})$, (f). It is shown in [DW 3 that $\operatorname{dim}_{G} X \leq \max \left(\operatorname{dim}_{G} A, 2\right)+\max \left(\operatorname{dim}_{G} B, 2\right)+1$ if $G=\mathbb{Q}, \mathbb{Z}_{p}, \mathbb{Z}_{l}$ or $\mathbb{Z} / p$. Therefore, if $H=\mathbb{Q}, \mathbb{Z}_{p}, \mathbb{Z}_{l}$ or $\mathbb{Z} / p$, then

$$
\begin{aligned}
\operatorname{dim}_{H} X & \leq \max \left(\operatorname{dim}_{H} A, 2\right)+\max \left(\operatorname{dim}_{H} B, 2\right)+1 \\
& \leq \max \left(\operatorname{dim}_{G} A, 2\right)+1+\max \left(\operatorname{dim}_{G} B, 2\right)+1+1
\end{aligned}
$$

(by Theorem $\mathrm{B}(\mathrm{f}))$. If $H=\mathbb{Z} / p^{\infty}$, then

$$
\begin{aligned}
\operatorname{dim}_{\mathbb{Z} / p^{\infty}} X & \leq \operatorname{dim}_{\mathbb{Z} / p} X \\
& \leq \max \left(\operatorname{dim}_{\mathbb{Z} / p} A, 2\right)+\max \left(\operatorname{dim}_{\mathbb{Z} / p} B, 2\right)+1 \\
& \leq \max \left(\operatorname{dim}_{\mathbb{Z} / p^{\infty}} A, 2\right)+1+\max \left(\operatorname{dim}_{\mathbb{Z} / p^{\infty}} B, 2\right)+1+1 \\
& \leq \max \left(\operatorname{dim}_{\text {Tor } G} A, 2\right)+\max \left(\operatorname{dim}_{\text {Tor } G} B, 2\right)+3 \\
& \leq \max \left(\operatorname{dim}_{G} A, 2\right)+\max \left(\operatorname{dim}_{G} B, 2\right)+3 .
\end{aligned}
$$

Remark. Theorem $\mathrm{C}$ is related to Problem 10 of [Ku]: Does $\operatorname{dim}_{G}(A \cup B) \leq$ $\operatorname{dim}_{G} A+\operatorname{dim}_{G} B+1$ hold for hereditarily paracompact $A \cup B$ ? Its solution for metrizable spaces and $G=\mathbb{Z}$ was announced by L. Rubin [R]. It is shown in $\left[\mathrm{D}-\mathrm{W}_{3}\right]$, that

$$
\operatorname{dim}_{G}(A \cup B) \leq \max \left(\operatorname{dim}_{G} A, 2\right)+\max \left(\operatorname{dim}_{G} B, 2\right)+1
$$

if $G=\mathbb{Q}, \mathbb{Z}_{l}$ or $\mathbb{Z} / p$ and $A \cup B$ is metrizable.

Theorem D. Suppose $f: X \rightarrow Y$ is a proper map of metrizable spaces and $G$ is an abelian group.

(1) If for each $k \leq \operatorname{dim}_{G} X+1$ all the groups $\left\{\check{H}^{k}\left(f^{-1}(y) ; \mathbb{Z}\right) \mid y \in Y\right\}$ are isomorphic and finitely generated, then

$$
\operatorname{dim}_{G} Y \leq \operatorname{dim}_{G} X+1,
$$


(2) if $\operatorname{dim}_{G} X$ is finite and for each $k \geq 0$ there are only finitely many isomorphic classes among $\left\{\check{H}^{k}\left(f^{-1}(y) ; \mathbb{Z}\right) \mid y \in Y\right\}$ and all these groups are finitely generated, then $\operatorname{dim}_{G} Y$ is finite.

Proof. Let $\delta(G)$ be obtained from $\sigma(G) \cup\left\{\mathbb{Z}_{l}\right\}$ by replacing each $\mathbb{Z} / p^{\infty}$ with $\mathbb{Z} / p$. Thus, $\delta(G)$ consists of principal ideal domains only. Let

$$
n=\max \left\{\operatorname{dim}_{H} X \mid H \in \delta(G)\right\}
$$

which is at most $\operatorname{dim}_{G} X+1$ (see Theorem $\mathrm{B}(\mathrm{e}),(\mathrm{f})$ and Bockstein Inequality (1).

(1) Notice that for each pair of points $y, z \in Y, \check{H}^{k}\left(f^{-1}(y) ; H\right)$ and $\check{H}^{k}\left(f^{-1}(z) ; H\right)$ are isomorphic finitely generated $H$-modules for all $k$. Indeed, if $H$ is torsion-free, then $\check{H}^{k}\left(f^{-1}(y) ; H\right) \approx \check{H}^{k}\left(f^{-1}(y) ; \mathbb{Z}\right) \otimes H$ and $\check{H}^{k}\left(f^{-1}(z) ; H\right) \approx \check{H}^{k}\left(f^{-1}(z) ; \mathbb{Z}\right) \otimes H$ as $H$ modules. If $H=\mathbb{Z} / p$, a field, then each of

$$
0 \rightarrow \check{H}^{k}\left(f^{-1}(y) ; \mathbb{Z}\right) \otimes H \rightarrow \check{H}^{k}\left(f^{-1}(y) ; H\right) \rightarrow \check{H}^{k+1}\left(f^{-1}(y) ; \mathbb{Z}\right) * H \rightarrow 0
$$

and

$$
0 \rightarrow \check{H}^{k}\left(f^{-1}(z) ; \mathbb{Z}\right) \otimes H \rightarrow \check{H}^{k}\left(f^{-1}(z) ; H\right) \rightarrow \check{H}^{k+1}\left(f^{-1}(z) ; \mathbb{Z}\right) * H \rightarrow 0
$$

splits and $\check{H}^{k+1}\left(f^{-1}(z) ; \mathbb{Z}\right) *(\mathbb{Z} / p) \subset \check{H}^{k+1}\left(f^{-1}(z) ; \mathbb{Z}\right) *\left(\mathbb{Z} / p^{\infty}\right)$ is trivial if $k \geq \operatorname{dim}_{G} X+1$. Indeed,

$$
\begin{aligned}
0 & \rightarrow \check{H}^{k}\left(f^{-1}(z) ; \mathbb{Z}\right) \otimes\left(\mathbb{Z} / p^{\infty}\right) \rightarrow \check{H}^{k}\left(f^{-1}(z) ; \mathbb{Z} / p^{\infty}\right) \\
& \rightarrow \check{H}^{k+1}\left(f^{-1}(z) ; \mathbb{Z}\right) *\left(\mathbb{Z} / p^{\infty}\right) \rightarrow 0
\end{aligned}
$$

is exact and $\check{H}^{k}\left(f^{-1}(z) ; \mathbb{Z} / p^{\infty}\right)=0$ as $\operatorname{dim}_{\mathbb{Z} / p^{\infty}} X \leq \operatorname{dim}_{G} X$. This proves that $\check{H}^{k}\left(f^{-1}(y) ; H\right)$ and $\check{H}^{k}\left(f^{-1}(z) ; H\right)$ are isomorphic and finitely generated if $k \leq \operatorname{dim}_{G} X+1$. If $k \geq \operatorname{dim}_{G} X+2$, both $\breve{H}^{k}\left(f^{-1}(y) ; H\right)$ and $\breve{H}^{k}\left(f^{-1}(z) ; H\right)$ are trivial as $\operatorname{dim}_{H} X \leq \operatorname{dim}_{G} X+1$. Now, it is shown in [D-W 4 ] that $\operatorname{dim}_{H} Y \leq$ $\operatorname{dim}_{H} X$ (see $\left[\mathrm{D}-\mathrm{W}_{2}\right]$ for the case $H=\mathbb{Z}$ ) which completes the proof in view of $\operatorname{dim}_{H} X \leq \operatorname{dim}_{G} X+1$ (use Theorem B(e), (f)).

(2) Notice that $Y$ is the union $Y_{1} \cup \cdots \cup Y_{m}$ of finitely many of its subsets so that for each pair of points $y, z \in Y_{i}, \check{H}^{k}\left(f^{-1}(y) ; \mathbb{Z}\right)$ and $\check{H}^{k}\left(f^{-1}(z) ; \mathbb{Z}\right)$ are isomorphic if $k \leq \operatorname{dim}_{G} X+1$. By (1), $\operatorname{dim}_{G} Y_{i}$ is finite for each $i$, which is all we need in view of Theorem $C$.

4.1. Lemma. If $X \in A N E$ (metrizable spaces) is metrizable and $Y \in A E(X)$, then $K \in A E(X)$ for any $C W$ complex $K$ weakly homotopically equivalent to $Y$.

Proof. Let $\pi: K \rightarrow Y$ be a weak homotopy equivalence (i.e., a map inducing isomorphisms of all homotopy groups). The important property of $\pi$ is that $\pi_{*}:[Z, K] \rightarrow[Z, Y]$ is a bijection for all spaces $Z$ which are homotopy equivalent to $\mathrm{CW}$ complexes. Suppose $f: A \rightarrow K$ is a map and $A$ is a closed subset of $X$. Extend $f$ to $f^{\prime}: V \rightarrow K$, where $V$ is a closed neighborhood of $A$ in $X$ (see Theorem 2.2). Let $F: X \rightarrow Y$ be an extension of $\pi \circ f^{\prime}: V \rightarrow Y$ and choose $F^{\prime}: X \rightarrow K$ so that $F \approx \pi \circ F^{\prime}$ (such $F^{\prime}$ exists since all metrizable $X \in A N E$ (metrizable spaces) are homotopy equivalent to CW complexes-see [M-S, p. 317]). Then $\pi \circ F^{\prime} \mid$ int $V \approx F \mid$ int $V=\pi \circ f^{\prime} \mid$ int $V$, so $F^{\prime} \mid$ int $V \approx$ 
$f^{\prime} \mid$ int $V$ and $F^{\prime}\left|A \approx f^{\prime}\right| A=f$. Thus, $f$ extends over $X$ up to homotopy, so it extends over $X$ by the Homotopy Extension Theorem.

Theorem E. Suppose $X \in A N E$ (metrizable spaces) is metrizable and $G=$ $\prod_{s \in S} G_{s}$ is the direct product of abelian groups $\left\{G_{s}\right\}_{s \in S}$. Then

$$
\operatorname{dim}_{G} X=\max \left\{\operatorname{dim}_{G_{s}} X \mid s \in S\right\} .
$$

Proof. Clearly, $\operatorname{dim}_{G} X \geq \max \left\{\operatorname{dim}_{G_{s}} X \mid s \in S\right\}$ as each $G_{s}$ is a direct summand of $G$. Suppose $\max \left\{\operatorname{dim}_{G_{s}} X \mid s \in S\right\}=n$. Choose a CW complex $K_{s}$ of the type $K\left(G_{s}, n\right)$ for each $s \in S$. Clearly, the cartesian product $Y=\prod_{s \in S} K_{s} \in A E(X)$ as each $K_{s} \in A E(X)$ (see [H, Proposition 4.1 on p. 39]). Now, any CW complex $K$ weakly homotopically equivalent to $Y$ (for example, let $K$ be the geometric realization of the singular complex of $Y$ ) is of the type $K(G, n)$ and $K \in A E(X)$ by Lemma 4.1.

\subsection{Corollary. Suppose $X \in A N E$ (metrizable spaces) is metrizable. Then}

(a) $\operatorname{dim}_{\mathbb{Z} / p} X=\operatorname{dim}_{\widehat{\mathbb{Z}}_{p}} X$ for any prime $p$, where $\widehat{\mathbb{Z}}_{p}$ is the group of $p$-adic integers,

(b) if $X$ is compact, then $\operatorname{dim}_{\mathbb{Z} / p} X=\operatorname{dim}_{\mathbb{Z}_{p}} X$ for any prime $p$,

(c) $\operatorname{dim}_{G} X \geq \operatorname{dim}_{\mathbb{Q}} X$ for any abelian group $G \neq 0$.

Proof. In view of Corollary 3.1 it suffices to show $\operatorname{dim}_{\mathbb{Z} / p} X \geq \operatorname{dim}_{\widehat{\mathbb{Z}}_{p}} X\left(\widehat{\mathbb{Z}}_{p} \otimes\right.$ $(\mathbb{Z} / p)=\widehat{\mathbb{Z}}_{p} / p \cdot \widehat{\mathbb{Z}}_{p} \neq 0$ and $\left.\mathbb{Z} / p \in \sigma\left(\widehat{\mathbb{Z}}_{p} / p \cdot \widehat{\mathbb{Z}}_{p}\right)\right)$. Let $A=(\mathbb{Z} / p) \times\left(\mathbb{Z} / p^{2}\right) \times$ $\left(\mathbb{Z} / p^{3}\right) \cdots$ be the countable direct product. Notice that $A /$ Tor $A$ is not divisible by $p$. Indeed, if $([1],[1], \ldots)=p \cdot\left(\left[x_{1}\right],\left[x_{2}\right], \ldots\right)+\left(\left[t_{1}\right],\left[t_{2}\right], \ldots\right)$, where $p^{k} \cdot\left(\left[t_{1}\right],\left[t_{2}\right], \ldots\right)=(0,0, \ldots)$ for some $k \geq 1$, then there are numbers $y_{n} \in \mathbb{Z}$ such that $p^{k} \cdot t_{n}=p^{n} \cdot y_{n}$ for all $n \geq 1$. Hence, $t_{n}=p^{n-k} \cdot y_{n}$ and $[1]=p \cdot\left[x_{n}\right]+\left[t_{n}\right]$ is divisible by $p$ in $\mathbb{Z} / p^{n}$ for $n>k$, a contradiction. Thus, $\operatorname{dim}_{\mathbf{Z} / p} X=\operatorname{dim}_{A} X \geq \operatorname{dim}_{\widehat{\mathbf{Z}}_{p}} X$ by Theorem B(d) and Theorem E.

(b) Follows from (a) and the Bockstein Theorem (in view of $\sigma\left(\widehat{\mathbb{Z}}_{p}\right)=\left\{\mathbb{Q}, \mathbb{Z}_{p}\right\}$ ).

(c) In view of Theorem $B(a)-(c)$ and (a) it suffices to consider the case $\mathbb{Z} / p^{\infty} \in \sigma(G)$ for some prime $p$. Notice that the countable direct product $A=\left(\mathbb{Z} / p^{\infty}\right) \times\left(\mathbb{Z} / p^{\infty}\right) \times \cdots$ is not a torsion group (consider $\left(c_{1}, c_{2}, \ldots\right)$, where $c_{n}$ is of order $\left.p^{n}\right)$. Hence $\operatorname{dim}_{\mathbb{Q}} X \leq \operatorname{dim}_{A} X=\operatorname{dim}_{\mathbb{Z} / p^{\infty}} X \leq \operatorname{dim}_{G} X$.

Remark. Parts (b) and (c) of Corollary 4.2 were proved in [Ku, p. 25] (see also [Dy]) for locally compact spaces which are cohomologically locally connected, by using a different method based on the Universal Coefficient Formula.

Theorem F. Suppose $X$ is metrizable and $K$ is a connected $C W$ complex. If $K$ is simply connected, then the following conditions are equivalent:

(a) $\operatorname{dim}_{\pi_{m}(K)} X \leq m$ for each $m \geq 1$,

(b) $\operatorname{dim}_{H_{m}(K)} X \leq m$ for each $m \geq 1$.

Proof. For brevity, let $\mathscr{C}(X, m)$ be the class of all abelian groups $G$ such that $\operatorname{dim}_{G} X \leq m$. Let $n \geq 2$. Suppose $\pi_{m}(K) \in \mathscr{C}(X, m)$ if $n>m \geq 1$. Let $l=$ $\left\{p \mid \mathbb{Z} / p^{\infty} \in \mathscr{C}(X, n-1)\right\}$. Let $M$ be obtained from $K$ by attaching $(n+i)$-cells, $i \geq 1$, so that $\pi_{n+j}(M)=0$ for $j \geq 0$. Then $\pi_{n+1}(M, K) \approx \pi_{n}(K)$ (as $0=$ $\pi_{n+1}(M) \rightarrow \pi_{n+1}(M, K) \rightarrow \pi_{n}(K) \rightarrow \pi_{n}(M)=0$ is exact) and $\pi_{n+1}(M, K) \approx$ $H_{n+1}(M, K ; \mathbb{Z})$ by the Hurewicz Theorem. Thus, replacing $H_{n+1}(M, K ; \mathbb{Z})$ by $\pi_{n}(K)$ in the homology exact sequence, the following sequence is exact:

$$
H_{n+1}(K ; \mathbb{Z}) \rightarrow H_{n+1}(M ; \mathbb{Z}) \rightarrow \pi_{n}(K) \rightarrow H_{n}(K ; \mathbb{Z}) \rightarrow H_{n}(M ; \mathbb{Z}) \rightarrow 0 \text {. }
$$


If $q \notin l$, then $q$ - $\operatorname{Tor}\left(\pi_{m}(K)\right)=0$ (otherwise, $\mathbb{Z} / q^{\infty} \in \mathscr{C}(X, n-1)$, i.e. $q \in l)$. It follows from the exactness of $0 \rightarrow \pi_{m}(M) \otimes(\mathbb{Z} / q) \rightarrow \pi_{m}(M ; \mathbb{Z} / q) \rightarrow$ $\pi_{m}(M) *(\mathbb{Z} / q) \rightarrow 0$ (see $\left.[\mathrm{N}]\right)$ that $\pi_{m}(M ; \mathbb{Z} / q)=0$ for all $m$. Hence, $H_{m}(M ; \mathbb{Z} / q)=0$ for all $m($ see the $\bmod q$ Hurewicz Theorem in $[\mathrm{N}])$ and $H_{m}(M ; \mathbb{Z}) \otimes(\mathbb{Z} / q)=q-\operatorname{Tor}\left(H_{m}(M ; \mathbb{Z})\right)=0$ for all $m$.

Case I. $\mathbb{Q} \notin \mathscr{C}(X, n-1)$.

Now, $\pi_{m}(K)$ are torsion groups for all $m<n$ by Theorem B(c). Hence, each $H_{m}(M ; \mathbb{Z})$ is a torsion group as all $\pi_{m}(M)$ are torsion groups (see [S, p. 508]). By the exactness of $H_{n+1}(M ; \mathbb{Z}) \rightarrow \pi_{n}(K) \rightarrow H_{n}(K ; \mathbb{Z}) \rightarrow H_{n}(M ; \mathbb{Z}) \rightarrow 0$ we conclude $q-\operatorname{Tor}\left(\pi_{n}(K)\right) \approx q-\operatorname{Tor}\left(H_{n}(K ; \mathbb{Z})\right)$ for $q \notin l$ and

$$
\pi_{n}(K) /\left(\operatorname{Tor} \pi_{n}(K)\right) \approx H_{n}(K / \mathbb{Z}) /\left(\operatorname{Tor} H_{n}(K ; \mathbb{Z})\right) .
$$

If $\pi_{n}(K) \in \mathscr{C}(X, n)$ (resp. $\left.H_{n}(K ; \mathbb{Z}) \in \mathscr{C}(X, n)\right)$, then $\pi_{n}(K) /\left(\right.$ Tor $\left.\pi_{n}(K)\right)$ $\approx H_{n}(K ; \mathbb{Z}) /\left(\right.$ Tor $\left.H_{n}(K ; \mathbb{Z})\right) \in \mathscr{C}(X, n)$ by Theorem $\mathrm{B}(\mathrm{b})$. If $q \notin l$, then $q-\operatorname{Tor}\left(\pi_{n}(K)\right) \approx q-\operatorname{Tor}\left(H_{n}(K ; \mathbb{Z})\right) \in \mathscr{C}(X, n)$. If $p \in l$, then $\mathbb{Z} / p^{\infty} \in$ $\mathscr{C}(X, n-1)$ which implies $\mathbb{Z} / p \in \mathscr{C}(X, n)$ (see (1) of the Bockstein Inequality). Thus $p-\operatorname{Tor}\left(H_{n}(K ; \mathbb{Z})\right) \in \mathscr{C}(X, n)$ and $p-\operatorname{Tor}\left(\pi_{n}(K)\right) \in \mathscr{C}(X, n)$. Consequently, $\operatorname{Tor}\left(H_{n}(K ; \mathbb{Z})\right) \in \mathscr{C}(X, n)$ (resp. Tor $\left.\pi_{n}(K) \in \mathscr{C}(X, n)\right)$ by Theorem $\mathrm{B}(\mathrm{a})$ and, by Theorem $\mathrm{B}(\mathrm{b}), H_{n}(K ; \mathbb{Z}) \in \mathscr{C}(X, n) \quad\left(\right.$ resp. $\pi_{n}(K) \in$ $\mathscr{C}(X, n))$.

Case II. $\mathbb{Q} \in \mathscr{C}(X, n-1)$.

As $H_{m}(M ; \mathbb{Z}) \otimes(\mathbb{Z} / q)=q-\operatorname{Tor}\left(H_{m}(M ; \mathbb{Z})\right)=0$ for all $m$ and $q \notin l$, each $H_{m}(M ; \mathbb{Z})$ is a $\mathbb{Z}_{l}$-module (i.e., it is uniquely divisible by $\left.q \notin l\right)$. From the exactness of $0 \rightarrow \mathbb{Z}_{l} \rightarrow \mathbb{Q} \rightarrow \bigoplus_{p \in l} \mathbb{Z} / p^{\infty} \rightarrow 0$ we conclude (using Bockstein Inequality (c)) that $\mathbb{Z}_{l} \in \mathscr{C}(X, n)$. Let $A$ be the image of $H_{n+1}(M ; \mathbb{Z}) \rightarrow \pi_{n}(K)$. If $q \notin l$, then $q$-Tor $A$ is divisible by $q$ and is contained in $q$ - $\operatorname{Tor}\left(\pi_{n}(K)\right)$. Thus, if $q$-Tor $A \neq 0, \sigma(q$-Tor $A)=\left\{\mathbb{Z} / q^{\infty}\right\}$.

If $\pi_{n}(K) \in \mathscr{C}(X, n)$, then $q$-Tor $A \in \mathscr{C}(X, n)$ as $\sigma\left(q-\operatorname{Tor}\left(\pi_{n}(K)\right)\right) \subset$ $\mathscr{C}(X, n)$. If $p \in l$, then $\mathbb{Z} / p^{\infty} \in \mathscr{C}(X, n-1)$ which implies $\mathbb{Z} / p \in \mathscr{C}(X, n)$ (see Bockstein Inequality (1)). Thus $p$-Tor $A \in \mathscr{C}(X, n)$ which implies Tor $A \in$ $\mathscr{C}(X, n)$ by Theorem $\mathrm{B}(\mathrm{a})$. Since $A /$ Tor $A$ is divisible by all $q \notin l$, it is a $\mathbb{Z}_{l}$-module and $A /$ Tor $A \in \mathscr{C}(X, n)$ by Corollary 2.8. By Theorem $\mathrm{B}(\mathrm{b})$, $A \in \mathscr{C}(X, n)$. Hence $\pi_{n}(K) / A \in \mathscr{C}(X, n)$ and $H_{n}(K ; \mathbb{Z}) \in \mathscr{C}(X, n)$ (use Bockstein Inequality (a) in both cases).

Suppose $H_{n}(K ; \mathbb{Z}) \in \mathscr{C}(X, n)$ and $H_{n+1}(K ; \mathbb{Z}) \in \mathscr{C}(X, n+1)$. First we are going to show that $\operatorname{Tor}\left(\pi_{n}(K)\right) \in \mathscr{C}(X, n)$. As above, $p$ - $\operatorname{Tor}\left(\pi_{n}(K)\right) \in$ $\mathscr{C}(X, n)$ if $p \in l$. Suppose $q \notin l$. Let $\pi_{n}(K) \rightarrow H_{n}(K ; \mathbb{Z})$ be denoted by $\mu$. Given $\alpha \in q-\operatorname{Tor}\left(H_{n}(K ; \mathbb{Z})\right)$ there is $\beta \in \pi_{n}(K)$ with $\alpha=\mu(\beta)$ as $q-\operatorname{Tor}\left(H_{n}(M ; \mathbb{Z})\right)=0$. Let $k \geq 1$ be an integer so that $q^{k} \cdot \alpha=0$. Then, $q^{k} \cdot \beta \in A$ and there is $\beta^{\prime} \in A$ with $q^{k} \cdot \beta^{\prime}=q^{k} \cdot \beta$ as $A$ is divisible by $q$. Now, $\beta-\beta^{\prime} \in q$-Tor $\left(\pi_{n}(K)\right)$ and $\mu\left(\beta-\beta^{\prime}\right)=\alpha$. That demonstrates the exactness of

$$
0 \rightarrow q \text { - Tor } A \rightarrow q-\operatorname{Tor}\left(\pi_{n}(K)\right) \rightarrow q-\operatorname{Tor}\left(H_{n}(K ; \mathbb{Z})\right) \rightarrow 0 .
$$

If $\sigma\left(q-\operatorname{Tor}\left(H_{n}(K ; \mathbb{Z})\right)\right)=\{\mathbb{Z} / q\}$, then $q-\operatorname{Tor}\left(\pi_{n}(K)\right) \in \mathscr{C}(X, n)$ by Corollary 2.8. If $\sigma\left(q-\operatorname{Tor}\left(H_{n}(K ; \mathbb{Z})\right)\right)=\left\{\mathbb{Z} / q^{\infty}\right\}$ then $q$ - $\operatorname{Tor}\left(\pi_{n}(K)\right) \in \mathscr{C}(X, n)$ as $\sigma(q-\operatorname{Tor} A)=\left\{\mathbb{Z} / q^{\infty}\right\}$. So, the only remaining case is $q$ - $\operatorname{Tor}\left(H_{n}(K ; \mathbb{Z})\right)=0$, 
$q$-Tor $A \neq 0$. Notice that $H_{n+1}(K ; \mathbb{Z}) /\left(q-\operatorname{Tor}\left(H_{n+1}(K ; \mathbb{Z})\right)\right)$ cannot be divisible by $q$ in such a case. Indeed, $H_{n+1}(K ; \mathbb{Z}) /\left(q-\operatorname{Tor}\left(H_{n+1}(K ; \mathbb{Z})\right)\right)$ maps onto $\operatorname{ker}\left(H_{n+1}(M ; \mathbb{Z}) \rightarrow A\right)$ which would make $\operatorname{ker}\left(H_{n+1}(M ; \mathbb{Z}) \rightarrow A\right)$ a $\mathbb{Z}\left[\frac{1}{q}\right]$ module. Since $H_{n+1}(M ; \mathbb{Z})$ is a $\mathbb{Z}\left[\frac{1}{q}\right]$-module, $A$ would be a $\mathbb{Z}\left[\frac{1}{q}\right]$-module contradicting $q$-Tor $A \neq 0$. Thus, $H_{n+1}(K ; \mathbb{Z}) /\left(\operatorname{Tor}\left(H_{n+1}(K ; \mathbb{Z})\right)\right)$ is not divisible by $q$, i.e. $\mathbb{Z}_{q} \in \sigma\left(H_{n+1}(K ; \mathbb{Z})\right)$. By Theorem B(e), $\mathbb{Z}_{q} \in \mathscr{C}(X, n+1)$ and by the Bockstein Inequality (4), $\mathbb{Z} / q^{\infty} \in \mathscr{C}(X, n)$ which implies $q$ - $\operatorname{Tor}\left(\pi_{n}(K)\right) \in$ $\mathscr{C}(X, n)$ as $\sigma\left(q-\operatorname{Tor}\left(\pi_{n}(K)\right)\right)=\sigma(q-\operatorname{Tor} A)=\left\{\mathbb{Z} / q^{\infty}\right\}$.

Our second step is to show that $\pi_{n}(K) / \operatorname{Tor}\left(\pi_{n}(K)\right)$ is a $\mathbb{Z}_{l}$-module which implies $\pi_{n}(K) / \operatorname{Tor}\left(\pi_{n}(K)\right) \in \mathscr{C}(X, n)$. Let $l^{\prime}=$ all primes $\}-l$. As

$$
l^{\prime}-\operatorname{Tor}\left(H_{n}(M ; \mathbb{Z})\right)=0
$$

and $l^{\prime}-\operatorname{Tor}\left(\pi_{n}(K)\right) \rightarrow l^{\prime}-\operatorname{Tor}\left(H_{n}(K ; \mathbb{Z})\right)$ is an epimorphism, we get that the sequence

$$
\begin{aligned}
0 & \rightarrow A /\left(l^{\prime}-\text { Tor } A\right) \rightarrow \pi_{n}(K) /\left(l^{\prime}-\operatorname{Tor}\left(\pi_{n}(K)\right)\right. \\
& \rightarrow H_{n}(K) /\left(l^{\prime}-\operatorname{Tor}\left(H_{n}(K)\right)\right) \rightarrow H_{n}(M) \rightarrow 0
\end{aligned}
$$

is exact. Since both $H_{n}(K) /\left(l^{\prime}-\operatorname{Tor}\left(H_{n}(K)\right)\right)$ and $H_{n}(M)$ are $\mathbb{Z}_{l}$-modules, the kernel of $H_{n}(K) /\left(l^{\prime}-\operatorname{Tor}\left(H_{n}(K)\right)\right) \rightarrow H_{n}(M)$ is a $\mathbb{Z}_{l}$-module which implies that $\pi_{n}(K) /\left(l^{\prime}-\operatorname{Tor}\left(\pi_{n}(K)\right)\right)$ is a $\mathbb{Z}_{l}$-module as $A /\left(l^{\prime}\right.$-Tor $\left.A\right)$ is a $\mathbb{Z}_{l}$-module. This completes the proof of Theorem $\mathrm{F}$ in view of Theorem $\mathrm{B}(\mathrm{b})$.

Remark. The equivalence $(\mathrm{a}) \leftrightarrow(\mathrm{b})$ is due to A. Dranishnikov [ $\left.\mathrm{D}_{2}\right]$ for compact spaces.

Theorem G. Suppose $X$ is metrizable and $K$ is a connected $C W$ complex such that $\operatorname{dim}_{\pi_{m}(K)} X \leq m$ for each $m \geq 1$. Then $K \in A E(X)$ in the following cases:

(a) $\pi_{m}(K)=0$ for $m$ sufficiently large,

(b) $\operatorname{dim} X<\infty$,

(c) $X \in A N E$ (metrizable spaces).

Proof. (a) The proof is by induction on $N(K)=\min \left\{k \mid \pi_{i} K=0\right.$ for $\left.i>k\right\}-$ $\max \left\{j \mid \pi_{i} K=0\right.$ for $\left.i<j\right\}$. If $N(K)=0$, then $K \in K(G, m)$ for some $m$ and $K \in A E(X)$. Assume Theorem $G$ holds for all $L$ such that $N(L) \leq n, n \geq 0$, and $K$ is a CW complex with $N(K)=n+1$. Put $m=\min \left\{k \mid \pi_{i} K=0\right.$ for $i>k\}$. Let $L$ be the $\mathrm{CW}$ complex obtained from $K$ by adding $n$-cells, $n>m$, so that the inclusion $K \rightarrow L$ induces isomorphisms of $i$ th homotopy groups for $i<m$ and $\pi_{i} L=0$ for $i \geq m$. Replace the inclusion $K \rightarrow L$ by a Hurewicz fibration $K^{\prime} \rightarrow L^{\prime}$, where $L^{\prime}$ is a simplicial complex homotopy equivalent to $L$. The fiber $F$ of $K^{\prime} \rightarrow L^{\prime}$ is of type $K\left(\pi_{m} K, m\right)$, so $F \in A E(X) \quad(F$ is homotopy equivalent to a $\mathrm{CW}$ complex by [M]). By Theorem 2.3, $K^{\prime} \in A E(X)$ as $L^{\prime} \in A E(X)$. Since $K$ is homotopy equivalent to $K^{\prime}, K \in A E(X)$.

(b) Let $m=\operatorname{dim} X$. Let $L$ be the CW complex obtained from $K$ by adding $n$-cells, $n>m$, so that the inclusion $K \rightarrow L$ induces isomorphisms of $i$ th homotopy groups for $i<m+2$ and $\pi_{i} L=0$ for $i \geq m+2$. By (a), $L \in A E(X)$. Given $f: A \rightarrow K, A$ closed in $X$, choose an extension $f^{\prime}: X \rightarrow L$ of $f: A \rightarrow L$. By Theorem 5.6 of [Dyd] there is $g: X \rightarrow K$, $g \approx f^{\prime}$ in $L$ and $g \mid A \approx f$. By the Homotopy Extension Theorem, $f$ extends over $X$, i.e., $K \in A E(X)$.

(c) For each $m \geq 1$ let $L_{m}$ be the CW complex obtained from $K$ by adding $n$-cells, $n>m$, so that the inclusion $i_{m}: K \rightarrow L_{m}$ induces isomorphism of $i$ th 
homotopy groups for $i \leq m$ and $\pi_{i} L=0$ for $i \geq m+1$. Notice that there are maps $p_{m, m+1}: L_{m+1} \rightarrow L_{m}$ extending id: $K \rightarrow K$. Replace each $p_{m, m+1}$ by a Hurewicz fibration $q_{m, m+1}: K_{m+1} \rightarrow K_{m}$, where $K_{m}$ is a metrizable space homotopy equivalent to $L_{m}$, by induction on $n$. Notice that the fiber of $q_{m, m+1}$ is of type $K\left(\pi_{m+1} K, m+1\right)$. Also, there are maps $q_{m}: K \rightarrow K_{m}$ inducing isomorphisms of $i$ th homotopy groups for $i \leq m$. Our plan is to show that $K^{\prime}=\operatorname{inv} \lim \left(q_{m, m+1}: K_{m+1} \rightarrow K_{m}\right) \in A E(X)$. Suppose $f_{m}: A \rightarrow K_{m}$ are maps from a closed subset $A$ of $X$ so that $q_{m, m+1} \circ f_{m+1}=f_{m}$ for $m \geq 1$. Let $F_{m}: X \rightarrow K_{m}$ be an extension of $f_{m}$ for some $m \geq 1$. By using Theorem 2.3 (replace $q_{m, m+1}: K_{m+1} \rightarrow K_{m}$ with an equivalent fibration whose base is a simplicial complex), there is $G: X \rightarrow K_{m+1}$ extending $f_{m+1}$ such that $q_{m, m+1} \circ G \approx F_{m}$ rel. $A$. Since $K_{m}$ is metrizable, there is a lift $F_{m+1}: X \rightarrow K_{m+1}$ of $f_{m+1}$ such that $q_{m, m+1} \circ F_{m+1}=F_{m}$ (see [Du, p. 397]). This shows that $K^{\prime} \in A E(X)$. Since $K^{\prime}$ is weakly homotopically equivalent to $K$ (see [Co]), $K \in A E(X)$ by Lemma 4.1 .

Remark. Theorem $\mathrm{G}(\mathrm{b})$ is due to A. Dranishnikov $\left[\mathrm{D}_{2}\right]$ for $X$ compact.

\section{OPEN PROBLEMS}

5.1. Problem. Does $\operatorname{dim}_{G} X=\max \left\{\operatorname{dim}_{H} X \mid H \in \sigma(G)\right\}$ hold for any abelian group $G$ and any metrizable space $X$ ? What if $G$ is countable?

5.2. Problem. Does $\operatorname{dim}_{G} X \geq \operatorname{dim}_{G^{\prime}} X$ hold if $X$ is a metrizable space and $G^{\prime}$ is a subgroup of $G$ so that $G / G^{\prime}$ is torsion-free and divisible? What if $G$ is countable?

Remarks. Problem 5.2 is a special case of the first part of Problem 6 in [Ku]. Namely, there $X$ is a paracompact space and $G / G^{\prime}$ is torsion-free. That problem is known to have a positive answer for compact spaces (see [Ku, Theorem 5]) which can be easily deduced from Corollary 2.10 . A positive solution to Problem 5.1 would imply a positive solution to Problem 5.2 in view of the following observation: if $G / G^{\prime}$ is torsion-free, then $\sigma\left(G^{\prime}\right) \subset \sigma(G)$. Indeed, Tor $G^{\prime}=$ Tor $G$ in such a case and $(G /$ Tor $G) /\left(G^{\prime}\right.$ Tor $\left.G^{\prime}\right) \approx G / G^{\prime}$ is torsionfree. Thus, if $G /$ Tor $G$ is divisible by $p$, so is $G^{\prime} /$ Tor $G^{\prime}$.

5.3. Problem. Does $\operatorname{dim}_{\widehat{\mathbb{Z}}_{p}} X=\operatorname{dim}_{\mathbb{Z}_{p}} X$ hold if $X$ is a metrizable space and $\widehat{\mathbb{Z}}_{p}$ is the group of $p$-adic integers (i.e., it is the completion of $\mathbb{Z}$ in the topology generated by $p^{k} \cdot \mathbb{Z}, k=1, \ldots$, as the system of neighborhoods of 0$)$ ? More generally, does $\operatorname{dim}_{\widehat{G}} X=\operatorname{dim}_{G} X$ hold if $X$ is a metrizable space, $G$ is torsionfree, nondivisible, and $\widehat{G}$ is the profinite completion of $G$ (see [Su]).

Remark. Problem 5.3 was posed in [Ku] (see Problem $7 \mathrm{~b}$ ) for paracompact spaces. It has a positive solution for compact spaces as $\sigma\left(\widehat{\mathbb{Z}}_{p}\right)=\left\{\mathbb{Q}, \mathbb{Z}_{p}\right\}$. Notice that 5.3 is a special case of both 5.1 and $5.2\left(\widehat{\mathbb{Z}}_{p} / \mathbb{Z}_{p}\right.$ is torsion-free and divisible). Also, in view of Theorem $B(d)$, a positive answer to Problem 5.3 for all prime $p$ would imply $\operatorname{dim}_{G} X \geq \max \left\{\operatorname{dim}_{H} X \mid H \in \sigma(G)\right\}$.

5.4. Problem. Does there exist a compact metrizable space $X \in A N E$ (metrizable spaces) such that $\operatorname{dim}_{\mathbb{Q}} X<\infty$ and $\operatorname{dim} X=\infty$ ?

Remark. Problem 5.4 is equivalent to Problem 2 of $\left[D_{1}\right]$. 
5.5. Problem. Characterize functions $m:\{\mathbb{Q}\} \cup \bigcup_{p}\left\{\mathbb{Z}_{p}, \mathbb{Z} / p, \mathbb{Z} / p^{\infty}\right\} \rightarrow \mathbb{Z}_{+}$ such that there is a compact metrizable space $X \in A N E$ (metrizable spaces) with $\operatorname{dim}_{H} X=m(H)$ for each $H \in\{\mathbb{Q}\} \cup \bigcup_{p}\left\{\mathbb{Z}_{p}, \mathbb{Z} / p, \mathbb{Z} / p^{\infty}\right\}$ ( $p$ is a prime).

Remark. Problem 5.5 was asked by A. Dranishnikov [D] (Problem 1). It was motivated by Dranishnikov's result that for any function

$$
m:\{\mathbb{Q}\} \cup \bigcup_{p}\left\{\mathbb{Z}_{p}, \mathbb{Z} / p, \mathbb{Z} / p^{\infty}\right\} \rightarrow \mathbb{Z}_{+}
$$

satisfying Bockstein Inequalities (1)-(5) there is a compact metrizable space $X$ with $\operatorname{dim}_{H} X=m(H)$ for each $H \in\{\mathbb{Q}\} \cup \bigcup_{p}\left\{\mathbb{Z}_{p}, \mathbb{Z} / p, \mathbb{Z} / p^{\infty}\right\}$ ( $p$ is a prime).

5.6. Problem. Suppose $X$ is a metrizable space and $G=\prod_{i=1}^{\infty} G_{i}$ is the direct product of torsion-free abelian groups $\left\{G_{i}\right\}_{i \geq 1}$. Does $\operatorname{dim}_{G} X=\max \left\{\operatorname{dim}_{G_{i}} X \mid\right.$ $i \geq 1\}$ hold?

Remark. Problem 5.6 has a positive solution if $X$ is compact as $\sigma\left(\prod_{i=1}^{\infty} G_{i}\right)=$ $\bigcup_{i=1}^{\infty} \sigma\left(G_{i}\right)$ if all $G_{i}, i \geq 1$, are torsion-free. See also Corollary 2.9.

\section{REFERENCES}

[B] M. F. Bockstein, Homological invariants of topological spaces. I, Trudy Moskov. Mat. Obshch. 5 (1956), 3-80. (Russian)

[C] H. Cohen, A cohomological definition of dimension for locally compact Hausdorff spaces, Duke Math. J. 21 (1954), 209-224.

[Co] J. M. Cohen, The homotopy groups of inverse limits, Proc. London Math. Soc. 27 (1973), 159-192.

[D $D_{1}$ A. N. Dranishnikov, Homological dimension theory, Uspekhi Mat. Nauk 43 (1988), 11-55. (Russian)

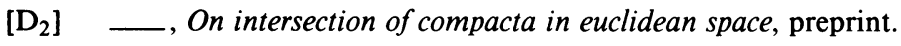

[Du] J. Dugundji, Topology, Allyn and Bacon, 1966.

[Dyd] J. Dydak, The Whitehead and Smale theorems in shape theory, Dissertationes Math. 156 (1979), 1-51.

[D- $\left.\mathrm{W}_{1}\right]$ J. Dydak and J. J. Walsh, Sheaves that are locally constant with applications to homology manifolds, Proceedings of the Geometric Topology and Shape Theory Conference held in Dubrovnik, Yugoslavia, September 29-October 10, 1986, Lecture Notes in Math., vol. 1283, Springer-Verlag, 1987, pp. 65-87.

$\left[\mathrm{D}-\mathrm{W}_{2}\right] \ldots$, Estimates of cohomological dimension of decomposition spaces, Topology Appl. 40 (1991), 203-219.

[D-W $\left.\mathrm{W}_{3}\right] \ldots$, Aspects of cohomological dimension for principal ideal domains, preprint.

$\left[\mathrm{D}-\mathrm{W}_{4}\right] \ldots$, Cohomology fibrations, preprint.

[Dy] E. Dyer, On the dimension of products, Fund. Math. 47 (1959), 141-160.

[F] L. Fuchs, Infinite abelian groups, Academic Press, New York and London, 1970.

[H] S. T. Hu, Theory of retracts, Wayne State Univ. Press, Detroit, Mich., 1965.

[Ko] Y. Kodama, Note on an absolute neighborhood extensor for metric spaces, J. Math. Soc. Japan 8 (1956), 206-215.

[Ku] W. I. Kuzminov, Homological dimension theory, Russian Math. Surveys 23 (1968), 1-45.

[Ma] H. R. Margolis, Spectra and the Steenrod algebra, North-Holland Mathematical Library, North-Holland, 1983.

[M] J. Milnor, On spaces having the homotopy type of a CW complex, Ann. of Math. (2) 65 (1959), 272-280.

[M-S] S. Mardešić and J. Segal, Shape theory, North-Holland, Amsterdam, 1982. 
[N] J. A. Neisendorfer, Primary homotopy theory, Mem. Amer. Math. Soc., no. 232, 1980.

[R] L. R. Rubin, Characterizing cohomological dimension: The cohomological dimension of $A \cup B$, Topology Appl. (to appear).

[S] E. Spanier, Algebraic topology, McGraw-Hill, New York, 1966.

[Su] D. Sullivan, Geometric topology, Part I: Localization, periodicity, and Galois symmetry, M.I.T. Press, 1970.

[Sz] T. Szele, On direct decompositions of abelian groups, J. London Math. Soc. 28 (1953), 247-250.

[Wa] J. J. Walsh, Dimension, cohomological dimension, and cell-like mappings, Lecture Notes in Math., vol. 870, Springer-Verlag, 1981, pp. 105-118.

[W] G. W. Whitehead, Elements of homotopy theory, Springer-Verlag, 1978.

Department of Mathematics, University of Tennessee, Knoxville, Tennessee 37996

E-mail address: dydak@utkvx.utk.edu 